“ (C) 2017 IEEE. Personal use of this material is permitted. Permission from IEEE must be obtained for all other uses, in any current or future media, including

reprinting/republishing this material for advertising or promotional purposes, creating new collective works, for resale or redistribution to servers or lists, or reuse of any copyrighted component of this work in other works." 


\section{Hybrid Power Plant Bidding Strategy Including a Commercial Compressed Air Energy Storage Aggregator and a Wind Power Producer}

\author{
Sahand Ghavidel, Mojtaba Jabbari Ghadi \\ Faculty of Engineering and \\ Information Technology, University of \\ Technology Sydney, PO Box 123, \\ Broadway, NSW 2007, Australia \\ \{sahand.ghavideljirsaraie,mojtaba.jabb \\ arighadi\}@student.uts.edu.au
}

\author{
Ali Azizivahed, Mostafa Barani, Jamshid \\ Aghaei \\ Department of Electrical and Electronics \\ Engineering, Shiraz University of \\ Technology, Shiraz, Iran \\ \{a.azizi,m.barani,aghaei\}@sutech.ac.ir
}

\author{
Li Li and Jiangfeng Zhang \\ Faculty of Engineering and \\ Information Technology, University of \\ Technology Sydney, PO Box 123, \\ Broadway, NSW 2007, Australia \\ \{li.li, Jiangfeng.Zhang\}@uts.edu.au
}

\begin{abstract}
In this paper, a commercial compressed air energy storage (CAES) aggregator equipped with a simple cycle mode operation having the ability to work like a gas turbine is coordinated with a wind power aggregator (WPA) as a hybrid power plant to participate in electricity markets. In the proposed approach, the WPA uses the CAES to tackle its stochastic input and uncertainties related to different electricity market prices, and CAES can also use WPA to manage its charging/discharging and simple cycle modes more economically. A three-stage stochastic decision-making method is used to model the mentioned optimization problem which considers three electricity markets including day-ahead, intraday and balancing markets. The problem is formulated as a mixed integer linear programming which can be solved with available commercial solvers. Also, conditional value-at-risk is added to the problem to control the financial risk of the problem and offer different operation strategies for different financials risk levels. The proposed method can provide both bidding quantity and bidding curves to be submitted to the electricity markets which is tested on a realistic case study based on a wind farm and electricity market located in Spain. The results confirm that the proposed method can provide extra profit in joint operation, have more flexibility and reduce the financial risks.
\end{abstract}

Index Terms-- Hybrid power plant, Wind power producer, Commercial compressed air energy storage, Conditional valueat-risk, Stochastic programming.

\section{INTRODUCTION}

Due to the stochastic, unstable and nondispatchable nature of wind generations, it is usually very difficult for these kinds of producers to participate in electricity markets and compete with other producers such as conventional power plants. For this reason, it is important to offer a new strategy for wind power aggregators (WPAs) to help them overcome these difficulties.

Nowadays, there are extensive attentions towards energy storage systems especially commercial compressed air energy storage (CAES) which is a mature energy storage system with the capability of functioning as a gas turbine when there is no air in the reservoir [1]. Commercial CAES facilities can provide an energy-shifting when there is instability in the electricity price. Besides, it is important to notice that the ability to work like a gas turbine (simple cycle mode operation) makes the CAES facilities to be different from other kinds of energy storages, because they can follow their daily schedule in a more optimized approach and exploit price spikes when the reservoir is entirely depleted [2].

To this end, a number of studies concentrate on best selfscheduling strategies for CAES facilities and calculate their energy arbitrage income in diverse electricity markets [3]. For example, a co-optimized CAES dispatch model to illustrate the significance of providing operating reserves and energy arbitrage in different U.S. electricity markets is presented in [4]. Ref. [2] proposes a risk-constrained bidding strategy for a commercial CAES plant that contributes in the day-ahead (DA) energy markets.

Many studies provide an offering strategy for WPAs to participate in electricity markets. In [5], a procedure is proposed to develop an offering strategy for a WPA including different types of electricity markets and considering the uncertainty related to stochastic nature of wind and prices of different markets. Ref. [6] recommends an offering strategy by considering a WPA as a price-maker in DA market. Ref. [7] studies an offering strategy of a WPA as a price-taker in the DA market and a price-maker in the balancing market. Ref. [8] presents a novel method considering the uncertainty related to the amount of wind power generation and load for corrective voltage control to cope with the states in which the power systems experience voltage instability due to severe contingencies.

Some studies provide offering strategies for WPAs along with other producers [9]. In this regard, Ref. [10] studies the joint operation of a WPA and a pumped-storage unit by considering the uncertainty related to fluctuating nature of wind and prices of market. The impact of wind uncertainty 


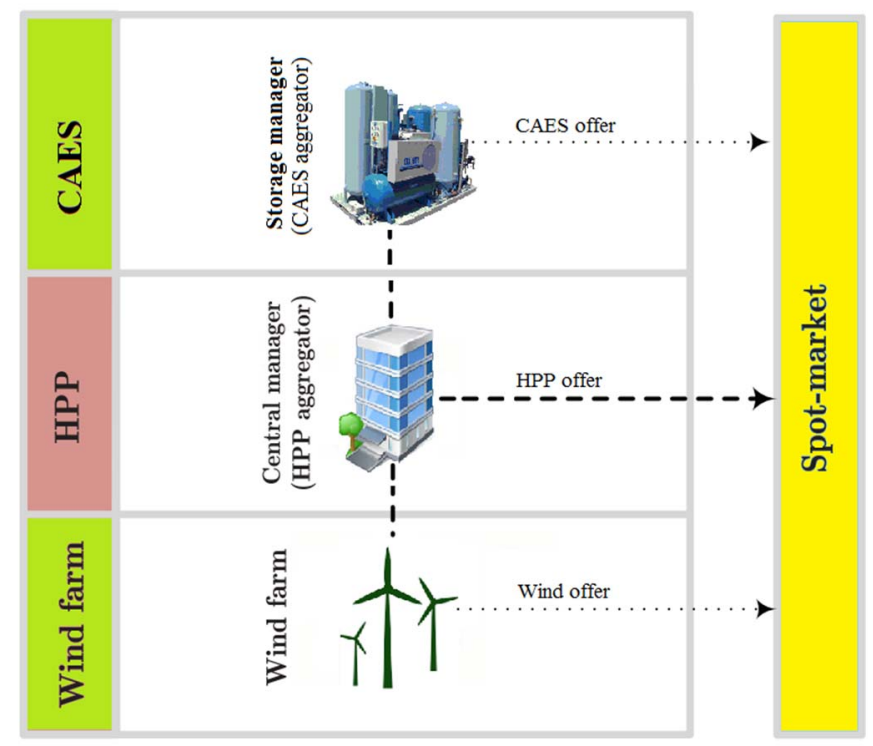

Fig. 1 Diagram illustration of three configurations.

on the amount of pumped-hydro stored energy in the future U.K. system is evaluated in [4]. Ref. [11] proposes a bidding strategies for a WPA and hydro facility to be able to participate in a DA market using conditional value-at-risk (CVar) model to control the financial risk. Ref. [12] evaluates two models including a WPA supported with a gas turbines, and WPA supported with a CAES. An offering strategy for a WPA and a flexible load which is able to cover the wind power imbalances is suggested in [13] for participation in a DA electricity market. In order to minimize the total operational cost including imbalance fines because of wind energy over/under-commitments, an optimal scheduling of critical peak pricing events is evaluated in [14] from the perspective of a demand response unit which has wind energy to be able to properly trade in the DA market. Ref. [15] presents a new offering strategy for a WPA to participate in three electricity markets including DA, intraday and balancing markets with the help of a demand response resource which is allowed to contribute to the intraday market.

This paper proposes an approach for the joint operation of a WPA and a commercial CAES aggregator as a hybrid power plant (HPP) in which the WPA utilizes the commercial CAES aggregator as a storage facility. The diagram illustration of three configurations (CAES only, WPA only and HPP) is shown in Fig. 1. The HPP participates in the DA, intraday and balancing markets. The uncertainties of wind generator production and market prices in three markets are considered. In order to find the best bidding strategy, the stochastic programming method is used, and then the CVar is added to control the financial risk.

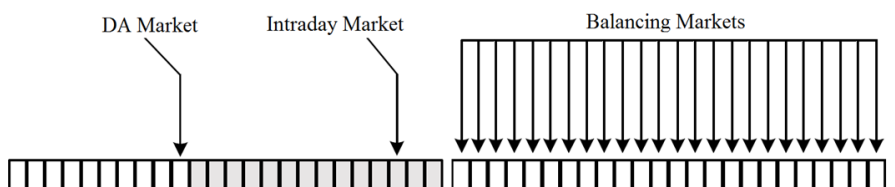

Current operating day: Day D Next operating day: Day $\mathrm{D}+1$

Fig. 2 Three electricity markets framework.

\section{PROBLEM FORMULATION}

In this section, the bidding strategy of the HPP is modeled as a profit maximization problem; in which the profit equals the revenue of the HPP minus its total cost. Three electricity markets including DA, intraday and balancing markets, as shown in Fig. 2, are considered in this paper [16]. Note that that the intraday market remains two and a half hours before the balancing market. Taking into consideration these three markets, the operational cost of CAES and the CVar, the objective function can be written as follows:

$$
\begin{aligned}
& \sum_{\omega=1}^{N_{\Omega}} \sum_{t=1}^{N_{T}} \rho_{\omega}\left[v_{t \omega}^{D} \cdot P_{t \omega}^{D, H p p}+v_{t \omega}^{I} \cdot P_{t \omega}^{I, H p p}+\right. \\
& \left.v_{t \omega}^{D} \cdot R_{t \omega}^{+} \cdot \delta_{t \omega}^{+, H p p}-v_{t \omega}^{D} \cdot R_{t \omega}^{-} \cdot \delta_{t \omega}^{-, H p p}-O C_{t \omega}\right]+ \\
& \beta\left(\zeta-\frac{1}{(1-\alpha)} \sum_{\omega=1}^{N_{\Omega}} \rho_{\omega} Z_{\omega}\right)
\end{aligned}
$$

Here, $\omega$ is index of scenario and $N_{\Omega}$ is the total number of scenarios. $t$ and $N_{T}$ are the index of time and the total period of time, respectively. $\rho_{\omega}$ is the probability of occurrence of each scenario. $v_{t \omega}^{D}$ and $v_{t \omega}^{I}$ are the DA and intra-day market prices, respectively. $P_{t \omega}^{D, H p p}$ and $P_{t \omega}^{I, H p p}$ are DA and intra-day offers of the HPP (superscript Hpp stands for hybrid power plant). $R_{t \omega}^{+}$and $R_{t \omega}^{-}$are the positive and negative imbalance price ratios. $\delta_{t \omega}^{+, H p p}$ and $\delta_{t \omega}^{-, H p p}$ are the positive and negative deviations of the generating power of HPP from the scheduled power. $O C_{t \omega}$ is the operational cost of CAES which is calculated based on the amount of power in charging/discharging and simple cycle modes, see equation (29). The last term of the equation (1) is related to modeling of the CVar. In this term, $\beta$ is the risk-aversion factor. By increasing the value of $\beta$, the financial risk will be decreased. $\zeta$ and $z_{\omega}$ are supplementary variables to calculate the CVar. Also, $\alpha$ is the confidence level.

The objective function defined in equation (1) is subject to some combined constraints related to both of WPA and commercial CAES provider and some other constraints specifically related to the CAES model.

The offer limitation of the HPP in the DA market can be written as follows:

$$
P_{t \omega}^{D, H p p}=P_{t \omega}^{D, \text { Wind }}+P_{t \omega}^{D, C A E S} \quad \forall t, \forall \omega
$$

where $P_{t \omega}^{D, \text { Wind }}$ and $P_{t \omega}^{D, C A E S}$ are the amount of wind and CAES powers offered to the DA markets, which are limited to the following constraints:

$$
\begin{aligned}
& 0 \leq P_{t \omega}^{D, \text { Wind }} \leq W^{\max } \quad \forall t, \forall \omega \\
& -P_{\text {Com }}^{\operatorname{Max}} \leq P_{t \omega}^{D, C A E S} \leq P_{E x p}^{\operatorname{Max}} \quad \forall t, \forall \omega
\end{aligned}
$$

where $W^{\max }$ is the WPA capacity, and $P_{E x p}^{\operatorname{Max}}$ and $P_{\text {com }}^{\operatorname{Max}}$ are the maximum expanding and compressing capacity of CAES, respectively. Note that the equation (2) can get negative values which means the HPP is considered to have the permission of both buying and selling in the DA market.

The HPP offer to the intra-day market is also limited as follows:

$$
P_{t \omega}^{I, H p p}=P_{t \omega}^{I, \text { Wind }}+P_{t \omega}^{I, C A E S} \quad \forall t, \forall \omega
$$

where $P_{t \omega}^{I, W i n d}$ and $P_{t \omega}^{I, C A E S}$ are the amount of wind and CAES powers offered to the intra-day markets. In fact, wind and 
CAES are not allowed to fully participate in the intra-day market. The amount of their participations are limited to a proportion of their offers to DA market or a percentage of their capacity [17] as follows:

$$
\begin{aligned}
& 0 \leq P_{t \omega}^{I, \text { Wind }} \leq \gamma \cdot P_{t \omega}^{D, \text { Wind }} \quad \forall t, \forall \omega \\
& -\gamma \cdot P_{\text {Com }}^{\text {Min }} \leq P_{t \omega}^{D, C A E S} \leq \gamma \cdot P_{E x p}^{\operatorname{Max}} \quad \forall t, \forall \omega
\end{aligned}
$$

where $\gamma$ is a bound factor for biddings to the intra-day market.

The total scheduled power of DA and intra-day offers can be formulated as follows:

$$
\begin{aligned}
& P_{t \omega}^{s c h, H p p}=P_{t \omega}^{D, H p p}+P_{t \omega}^{I, H p p} \quad \forall t, \forall \omega \\
& P_{t \omega}^{s c h, H p p}=P_{t \omega}^{s c h, \text { Wind }}+P_{t \omega}^{s c h, C A E S \quad \forall t, \forall \omega}
\end{aligned}
$$

where $P_{t \omega}^{s c h, H p p}$ is the scheduled power of HPP aggregator. $P_{t \omega}^{s c h, \text { Wind }}$ and $P_{t \omega}^{s c h, C A E S}$ are the amount of wind and CAES scheduled powers. Moreover, there are some constraints related to $P_{t \omega}^{s c h, \text { Wind }}$ and $P_{t \omega}^{s c h, C A E S}$ as follows:

$$
\begin{aligned}
& P_{t \omega}^{s c h, \text { Wind }}=P_{t \omega}^{D, \text { Wind }}+P_{t \omega}^{I, \text { Wind }} \quad \forall t, \forall \omega \\
& 0 \leq P_{t \omega}^{s c h, \text { Wind }} \leq W^{\max } \quad \forall t, \forall \omega \\
& -P_{\text {Com }}^{\operatorname{Max}} \leq P_{t \omega}^{s c h, C A E S} \leq P_{E x p}^{\operatorname{Max}} \quad \forall t, \forall \omega \\
& P_{t \omega}^{s c h, C A E S}=P_{t \omega}^{D, C A E S}+P_{t \omega}^{I, C A E S} \quad \forall t, \forall \omega \\
& P_{t \omega}^{s c h, C A E S}=P_{t \omega}^{s c h, D i s, C A E S}+P_{t \omega}^{s c h, S i m, C A E S}
\end{aligned}
$$

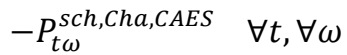

where $P_{t \omega}^{s c h, D i s, C A E S}, P_{t \omega}^{s c h, S i m, C A E S}$ and $P_{t \omega}^{s c h, C h a, C A E S}$ are the amount of discharging, simple cycle and charging powers scheduled for CAES. The limitations of CAES power in different modes of operation are as follows:

$$
\begin{array}{ll}
0 \leq P_{t \omega}^{s c h, D i s, C A E S} \leq P_{E x p}^{\operatorname{Max}} \cdot U_{t \omega}^{s c h, D i s, \text { CAES }} & \forall t, \forall \omega \\
0 \leq P_{t \omega}^{s c h, \text { SimCAES }} \leq P_{E x p}^{\operatorname{Max}} \cdot U_{t \omega}^{s c h, \text { Sim,CAES }} & \forall t, \forall \omega \\
0 \leq P_{t \omega}^{s c h, C h a, C A E S} \leq P_{\text {Com }}^{\operatorname{Max}} \cdot U_{t \omega}^{s c h, \text { Cha,CAES }} & \forall t, \forall \omega
\end{array}
$$

where $U_{t \omega}^{s c h, D i s, C A E S}, U_{t \omega}^{s c h, S i m, C A E S}$ and $U_{t \omega}^{s c h, D i s, C A E S}$ are the binary variables that show the operating status of the CAES (i.e. discharging, simple cycle or charging modes). Note that the CAES can only operate in one of the mentioned modes at each of time periods and scenarios. This concept can be mathematically formulated as the following constraint:

$$
U_{t \omega}^{s c h, D i s, C A E S}+U_{t \omega}^{s c h, S i m, C A E S}+U_{t \omega}^{s c h, C h a, C A E S} \leq 1 \quad \forall t, \forall \omega(18)
$$

The total negative and positive imbalances based on the scheduled power of HPP, real wind power production, and the power production of CAES can be defined as following constraints:

$$
\begin{aligned}
& \delta_{t \omega}^{H p p}=W_{t \omega}+C A E S_{t \omega}-P_{t \omega}^{s c h, H p p} \quad \forall t, \forall \omega \\
& \delta_{t \omega}^{H p p}=\delta_{t \omega}^{+, H p p}-\delta_{t \omega}^{-, H p p} \quad \forall t, \forall \omega \\
& 0 \leq \delta_{t \omega}^{+, H p p} \leq W_{t \omega}+C A E S_{t \omega} \quad \forall t, \forall \omega \\
& 0 \leq \delta_{t \omega}^{-, H p p} \leq W^{\max }+P_{E x p}^{M a x} \quad \forall t, \forall \omega
\end{aligned}
$$

where $W_{t \omega}$ and $C A E S_{t \omega}$ are the real wind power production and CAES power that will be finally delivered. In this paper, it is assumed that real power of CAES and its scheduled value are equal. In other words, it is assumed that there is no uncertainty in case of CAES power production.

The scheduled energy level of the CAES (i.e. also called state-transition equation) is defined as follows:

$$
\begin{aligned}
& E_{\omega}^{s c h, C A E S}(t)=E_{\omega}^{s c h, C A E S}(t-1) \\
& +\left(P_{\omega}^{s c h, C h a, C A E S}(t)\right. \\
& \left.-P_{\omega}^{s c h, D i s, C A E S}(t)\right) \times E R \\
& E_{C A E S}^{\operatorname{Min}} \leq E_{t \omega}^{s c h, C A E S} \leq E_{C A E S}^{\operatorname{Max}} \quad \forall t, \forall \omega
\end{aligned}
$$

where $E R$ is the energy rate ratio that converts value of power to the energy. $E_{C A E S}^{M i n}$ and $E_{C A E S}^{M a x}$ are the minimum and maximum amount of energy level that can be scheduled for the CAES, respectively.

For the calculation of risk, the following constraints are required:

$$
\begin{aligned}
& \quad-\sum_{t=1}^{N_{T}}\left[v_{t \omega}^{D} \cdot P_{t \omega}^{D, H p p}+v_{t \omega}^{I} P_{t \omega}^{I, H p p}+v_{t \omega}^{D} \cdot R_{t \omega}^{+} \cdot \delta_{t \omega}^{+, H p p}-\right. \\
& \left.v_{t \omega}^{D} \cdot R_{t \omega}^{-} \cdot \delta_{t \omega}^{-, H p p}\right]+\zeta-z_{\omega} \leq 0 \quad \forall \omega \\
& z_{\omega} \geq 0 \quad \forall \omega
\end{aligned}
$$

In order to propose non-decreasing curves to the DA market and applying them to the unpredictable conditions related to the decisions made in this market, the following constraints can be defined:

$$
\begin{aligned}
& \left(P_{t \omega}^{D, H p p}-P_{t \omega^{\prime}}^{D, H p p}\right) \cdot\left(v_{t \omega}^{D}-v_{t \omega^{\prime}}^{D}\right) \geq 0 \quad \forall t, \forall \omega, \forall \omega^{\prime} \\
& P_{t \omega}^{D, H p p}=P_{t \omega^{\prime}}^{D, H p p} \quad \forall t, \forall \omega, \forall \omega^{\prime}: v_{t \omega}^{D}=v_{t \omega^{\prime}}^{D}
\end{aligned}
$$

Finally, the equation related to operational cost of CAES can be written as follows:

$$
\begin{gathered}
O C_{t \omega}=P_{t \omega}^{s c h, D i s, C A E S} \times\left(H R_{\text {Dis }} \times N G_{\text {Price }}+V O M_{\text {Exp }}\right)+ \\
P_{t \omega}^{s c h, \text { Sim }, \text { CAES }} \times\left(H_{\text {Sim }} \times N G_{\text {Price }}+V O M_{E x p}+V O M_{\text {Com }}\right)+ \\
P_{t \omega}^{s c h, C h a, C A E S} \times\left(\text { VOM }_{\text {Com }}\right) \quad \forall t, \forall \omega
\end{gathered}
$$

where $H R_{\text {Dis }}$ and $H R_{\text {Sim }}$ are the heat rate in the discharging and simple cycle modes, respectively. $V O M_{E x p}$ and $V O M_{C o m}$ are the variable operation and maintenance cost for the expander and compressor of the CAES. $N G_{\text {Price }}$ refers to the natural gas price.

\section{RESULTS AND DISCUSSIONS}

The proposed methodology aims to coordinate a CAES aggregator with a WPA as a HPP to participate in electricity markets. The CAES is equipped with a simple cycle mode operation. Wind power and market price uncertainties are modelled and generated with a set of scenarios using an adapted hybrid neural network and improved particle swarm optimization [16].

The proposed method is applied on a real case study in the Sotavento wind farm located in Spain with 26.54 MW capacity [18]. The stochastic wind power generation is modelled using the procedure presented in [16]. In order to train the artificial neural network, the wind power historical data of year 2010 are used. The scenarios related to market prices are derived by a three-step process as presented in [19]. The historical data of market prices are also derived based on the Iberian Peninsula electricity market [20]. The uncertainties of the problem are modelled through a scenario 
tree with 3000 scenarios $(10 \times 5 \times 6 \times 10)$ including ten, five, six and, ten scenarios for DA, intraday, balancing market prices and the wind generation, respectively. The simulation results are presented for 12th of March, 2010.

The required heat rate of CAES for discharging mode is considered to be 0.4185 , and twice of this amount is considered for simple cycle mode. Natural gas price is equal to $3.5 € / G J$. Variable operation and maintenance cost of expander and compressor are equally considered to be 0.87 $€ / k W h$. Minimum and maximum levels of air storage in cavern are 1 and $15 \mathrm{MW}$, respectively. Also, the initial level of air stored in cavern is considered to be $1 \mathrm{MW}$. The energy ratio of CAES is equal to 0.95 .

The confidence level in the modelling of CVar is considered to be 0.95 . Moreover, the upper bound level of wind power production for adjustment market is considered to be 30 precent of its production level in DA market.

The proposed model is firstly implemented in MATLAB software for the scenario generation and reduction, and then the given data are imported to GAMS software by means of GAMS/MATLAB interface to solve the optimization problem. Note that the problem is solved using CPLEX solver, and the simulations are executed in 120.756 seconds on a $2.3 \mathrm{GHz}$ Intel ${ }^{\mathbb{B}} \mathrm{CORE}^{\mathrm{TM}} \mathrm{i} 5$ laptop with $8 \mathrm{~GB}$ of RAM.

In this paper, four cases are considered to evaluate applicability and effectiveness of the proposed approach. The base case does not consider the CAES simple cycle mode, financial risk and bidding curves. The second case considers bidding curve strategy. The third case considers the financial risk and the fourth case study considers the CAES simple cycle mode. These cases studies are provided in detail as follows.

\section{A. Case I: Base Case}

In this case, the WPA and CAES aggregator are coordinated to participate in the electricity markets without considering the CAES simple cycle mode, financial risk and bidding curves. In this regard, the risk factor $\beta$ is considered to be equal to zero. The CAES simple cycle mode is not added to the formulation. Also, equations (27) and (28) which are related to modeling bidding curves are not included in the optimization problem.

Fig. 3 shows the comparison of hourly energy bids/offers in the DA market for three different configurations including WPA only, CAES aggregator only, and HPP. As it can be seen, the CAES stores more energy during off-peak hours in the joint operation with the help of the WPA, and releases the stored energies to the market during the peak hours. Moreover, it is obvious from Fig. 3 that the HPP has more flexibility compared to two independent aggregators and can provide more power in some required periods of time such as peak hours. Fig. 4 shows the comparison of hourly expected profit under the three mentioned configurations. As it is shown, in some hours (e.g. hours 2 to 7), the hourly profit of HPP is less than the WPA. The reason is that the CAES is firstly trying to fully exploit the wind to charge its cavern,

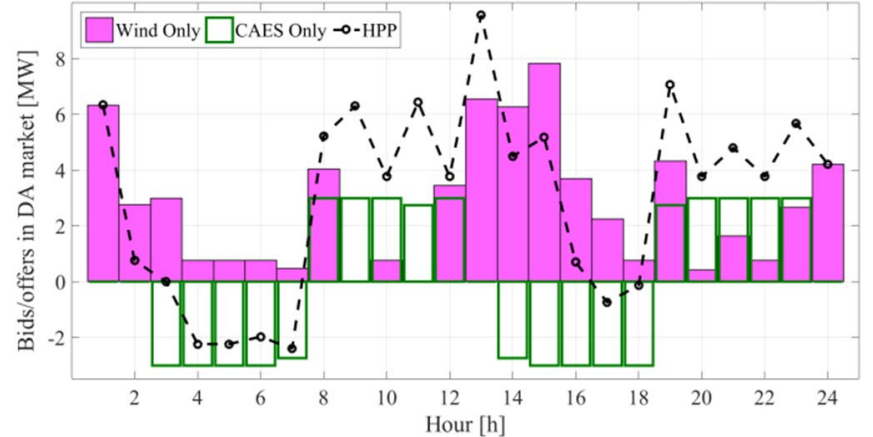

Fig. 3 Optimal energy hourly bids/offers in the DA market.

and then buy the rest of its needed energy from the markets. On the other hand, in some hours (e.g. hours 19 to 24), the hourly profit of HPP is more than the WPA due to the use of both the WPA and the CAES aggregator.

\section{B. Case II: Considering Bidding Curve}

In this case, the WPA and CAES aggregator are in coordination to participate in the markets with regard to bidding curves; while the CAES simple cycle mode and the financial risk are not considered. To this end, the risk factor $\beta$ is considered to be equal to zero. As in the previous case, the CAES simple cycle mode is not added to the formulation. But, equations (27) and (28) which are related to modeling bidding curves are included in the optimization problem.

Fig. 5 shows the optimal charging/discharging variations in the behaviour of CAES in the DA market for each scenario of the joint operation in Case II. It can be seen that by considering the bidding strategy formulation, the CAES power can adopt different patterns for tackling all scenarios related to the fluctuations of DA market.

Fig. 6 shows the results for bidding curves for seven sample hours (i.e. hours 4, 7, 10, 14, 15, 17 and 19) in the DA market. It can be seen that in the curve of hour 10, the HPP offers zero power to the market for the prices less than $27 €$. In the curve of hour 4 , for all prices, the HPP is bidding powers less than zero. In other words, in this situation, the HPP tries to only buy electricity from the DA market. The curve of hour 7 has the same pattern as that of hour 4, except offering zero power for prices more than $15 €$. In the bidding curve of hour 19, there are two types of pattern. In this hour, the HPP tries to buy electricity for the prices less than $21 €$, and sells electricity for the prices more than $21 €$. In the curve of hours 14,15 and 17, the HPP only sells power to the

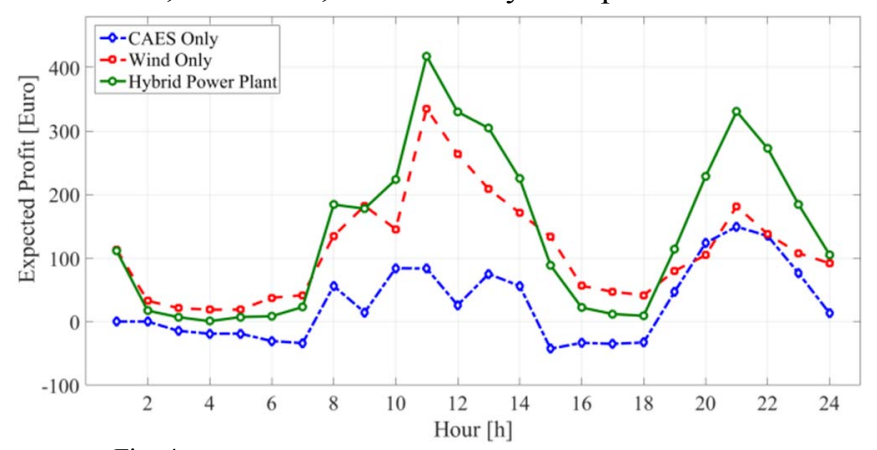

Fig. 4 Hourly expected profit for three different configurations. 


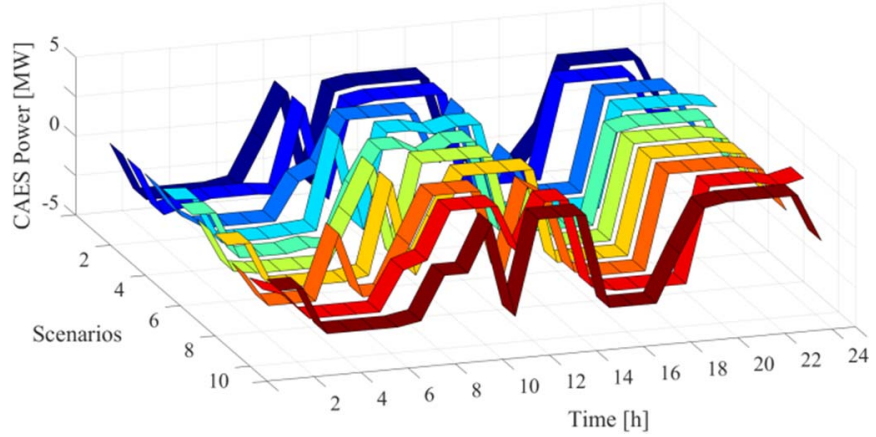

Fig. 5 Optimal charging/discharging variations in the behaviour of CAES.

market. Note that, in the hour 17 , the offering curve is only a straight line which means for all prices, the HPP offers the same amount of power to the market.

\section{Case III: Considering Financial Risk}

In this case, the WPA and CAES aggregator are coordinated to participate in the markets with considering bidding curves and financial risk while the simple cycle mode of CAES is not modelled. Similar to Case II, equations (27) and (28) are included to the optimization problem, and the CAES simple cycle mode is not added to the formulation. However, the risk factor $\beta$ is considered to vary from 0.1 to 1.

Fig. 7 and Fig. 8 show the changing pattern of different configurations including WPA only, CAES aggregator only, and HPP as well as the extra profit of using HPP compared to independent operations. As it can be seen in these figures, by increasing amount of the risk factor $\beta$, profits related to all the configurations are decreased. However, these decreases of the profits values are reasonable because the amount of financial risk in the system will be decreased as well. However, the extra profit of using HPP tends to increase with the increasing of $\beta$ value. In other words, by using the joint model, system can achieve more profit even by considering financial risk. Note that, the maximum extra profit (i.e. equal to 20.8317) is achieved at risk factor $\beta=0.7$, and the minimum extra profit is achieved at $\beta=0.2$. In fact, it shows the robustness of system operation in case of proposed join configuration versus increase of risk factor.

\section{A. Case IV: Considering CAES Simple Cycle Mode}

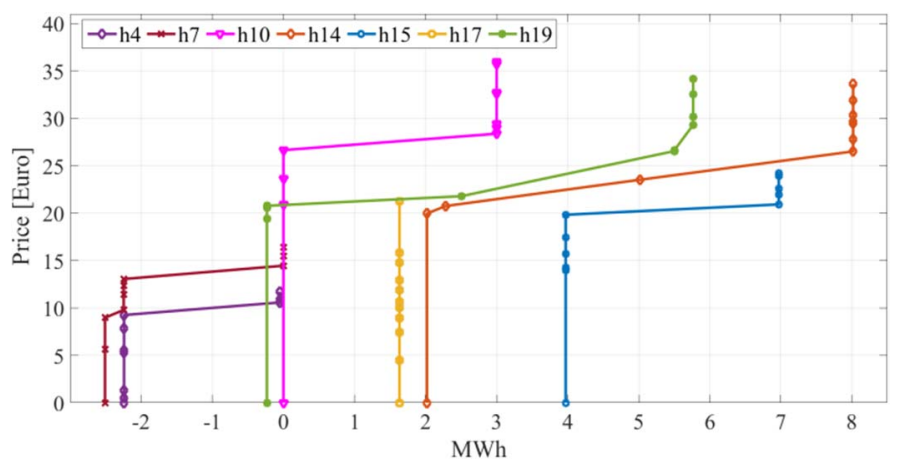

Fig. 6 Bidding curves of HPP in DA market.

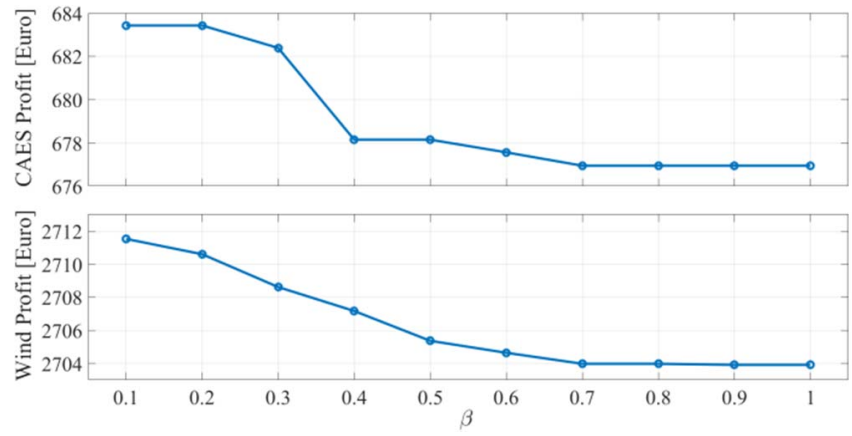

Fig. 7 Comparison of CAES and wind profit for different values of $\beta$.

In this scenario, the WPA and CAES aggregator are coordinated to participate in the markets with considering bidding curves, financial risk and the CAES simple cycle mode. Similar to Case II, equations (27) and (28) are included to the optimization problem. Also, the risk factor $\beta$ is considered to be 0.6 . Besides, the CAES simple cycle mode is added to the formulation.

Fig. 9 shows the comparison of hourly energy bids/offers of CAES to the DA market in the joint operation for two cases of with/without CAES simple cycle mode. As it can be illustrated, in the case of having CAES simple cycle mode, there are less hours of buying from the market. In other words, CAES does not need to charge the cavern as much as the situation in which there is no simple cycle mode. Also, in case of some hours e.g. 1 and 2 in which the cavern is in its initial values (i.e. it is assumed that the initial value of cavern is in its minimum level), the CAES can sell energy by using the simple cycle mode. This extra feature can be helpful for the whole system when there is a strike in the electricity price. For example, when the price of energy in a specific is high, there is no generating power for WPA and also the cavern is in its minimum level, the HPP can use the simple cycle mode of CAES and immediately sell energy to reach maximum profit of price fluctuations in the energy market.

Fig. 10 shows the comparison of energy level changes in the CAES cavern in the joint operation for two cases of with/without simple cycle mode of CAES. As it can be seen, in the case of utilizing CAES simple cycle mode, the energy level of cavern does not reach its maximum, which means the system would rather sell energy using CAES simple cycle mode in that particular hour (hour 6 as can be seen in the

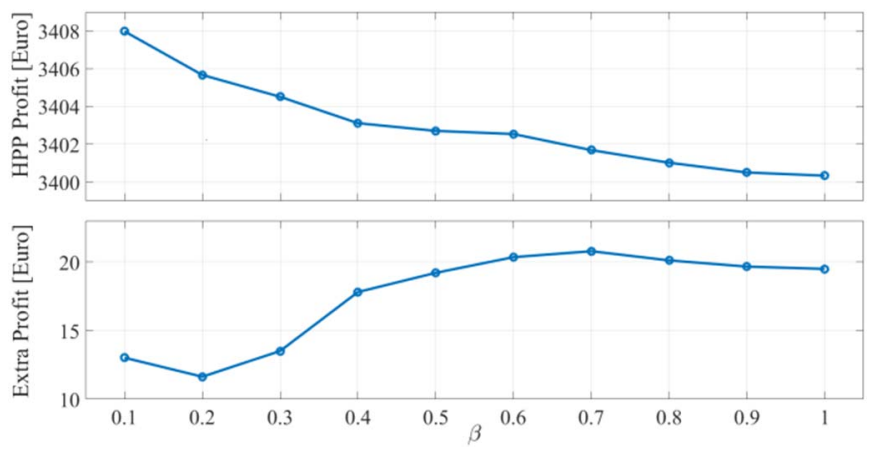

Fig. 8 Comparison of HPP profit and extra profit for different values of $\beta$. 


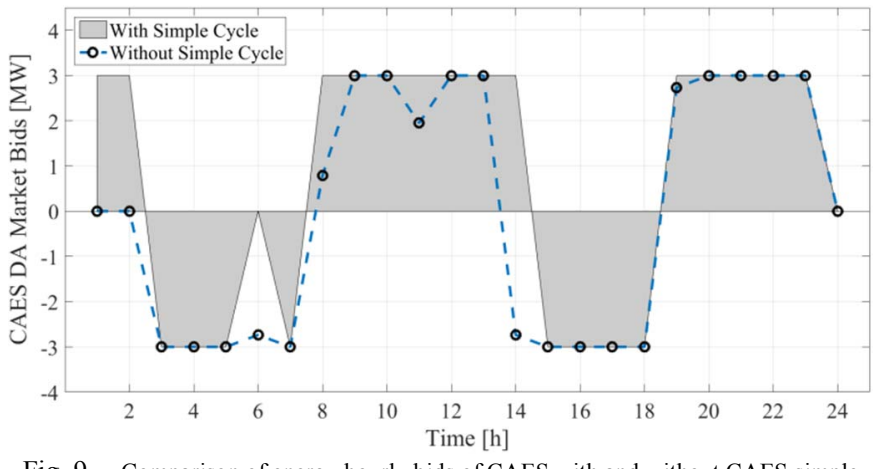

Fig. 9 Comparison of energy hourly bids of CAES with and without CAES simple cycle mode.

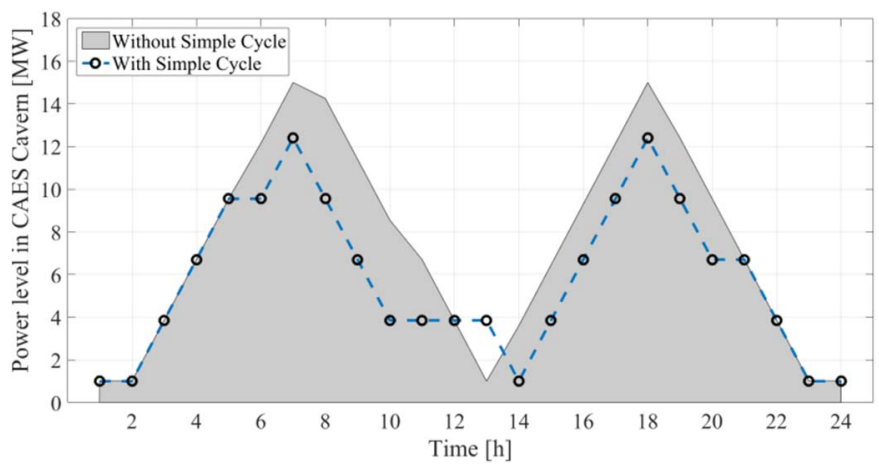

Fig. 10 Comparison of energy level change in the CAES cavern with and without CAES simple cycle mode.

figure) instead of buying it because of the more profitable tradeoff. Also, in some hours such as 13 and 14, there is a shift in cavern depletion by using the CAES simple cycle mode, which shows more flexibility in the system.

\section{CONCLUSION}

In this paper, the joint operation of a WPA and a CAES aggregator has been investigated. The problem has been formulated as a three-stage stochastic programing using mixed integer linear programming which can be solved with the available commercial solvers. Three electricity markets including DA, intraday and balancing markets have been considered.

The HPP can provide both energy quantity and bidding curves. In order to tackle the financial risk of the system, the CVar has been employed in the optimization problem. Moreover, a simple cycle mode operation has been considered in the CAES to increase the flexibility of the system in case of energy price strike.

\section{REFERENCES}

[1] E. Fertig and J. Apt, "Economics of compressed air energy storage to integrate wind power: A case study in ERCOT," Energy Policy, vol. 39, pp. 2330-2342, 2011

[2] S. Shafiee, H. Zareipour, A. M. Knight, N. Amjady, and B. Mohammadi-Ivatloo, "Risk-Constrained Bidding and Offering Strategy for a Merchant Compressed Air Energy Storage Plant," IEEE Transactions on Power Systems, 2016.

[3] S. Shafiee, H. Zareipour, A. M. Knight, N. Amjady, and B. Mohammadi-Ivatloo, "Risk-constrained bidding and offering strategy for a merchant compressed air energy storage plant," IEEE Transactions on Power Systems, vol. 32, pp. 946-957, 2017.
M. Black and G. Strbac, "Value of bulk energy storage for managing wind power fluctuations," IEEE transactions on energy conversion, vol. 22, pp. 197-205, 2007.

[5] J. M. Morales, A. J. Conejo, and J. Pérez-Ruiz, "Short-term trading for a wind power producer," IEEE Transactions on Power Systems, vol. 25, pp. 554-564, 2010.

[6] L. Baringo and A. J. Conejo, "Strategic offering for a wind power producer," IEEE Transactions on Power Systems, vol. 28, pp. 4645-4654, 2013.

[7] M. Zugno, J. M. Morales, P. Pinson, and H. Madsen, "Pool strategy of a price-maker wind power producer," IEEE Transactions on Power Systems, vol. 28, pp. 3440-3450, 2013.

[8] A. Rabiee, A. Soroudi, B. Mohammadi-Ivatloo, and M. Parniani, "Corrective voltage control scheme considering demand response and stochastic wind power," IEEE Transactions on Power Systems, vol. 29, pp. 2965-2973, 2014.

[9] M. Güçyetmez and E. Cam, "A new hybrid algorithm with geneticteaching learning optimization (G-TLBO) technique for optimizing of power flow in wind-thermal power systems," Electrical Engineering, vol. 98, pp. 145-157, 2016.

[10] J. Garcia-Gonzalez, R. M. R. de la Muela, L. M. Santos, and A. M. Gonzalez, "Stochastic joint optimization of wind generation and pumped-storage units in an electricity market," IEEE Transactions on Power Systems, vol. 23, pp. 460-468, 2008.

[11] A. A. S. de la Nieta, J. Contreras, and J. I. Munoz, "Optimal coordinated wind-hydro bidding strategies in day-ahead markets," IEEE Transactions on Power Systems, vol. 28, pp. 798-809, 2013.

[12] J. B. Greenblatt, S. Succar, D. C. Denkenberger, R. H. Williams, and R. H. Socolow, "Baseload wind energy: modeling the competition between gas turbines and compressed air energy storage for supplemental generation," Energy Policy, vol. 35, pp. 1474-1492, 2007.

[13] J. Mohammadi, A. Rahimi-Kian, and M.-S. Ghazizadeh, "Aggregated wind power and flexible load offering strategy," IET renewable power generation, vol. 5, pp. 439-447, 2011.

[14] X. Zhang, "Optimal scheduling of critical peak pricing considering wind commitment," IEEE Transactions on Sustainable Energy, vol. 5, pp. 637-645, 2014.

[15] E. Heydarian-Forushani, M. P. Moghaddam, M. K. Sheikh-ElEslami, M. Shafie-khah, and J. P. Catalão, "Risk-constrained offering strategy of wind power producers considering intraday demand response exchange," IEEE Transactions on sustainable energy, vol. 5, pp. 1036-1047, 2014.

[16] J. Aghaei, M. Barani, M. Shafie-Khah, A. A. S. de la Nieta, and J. P. Catalão, "Risk-constrained offering strategy for aggregated hybrid power plant including wind power producer and demand response provider," IEEE Transactions on Sustainable Energy, vol. 7, pp. 513-525, 2016.

[17] A. J. Conejo, M. Carrión, and J. M. Morales, Decision making under uncertainty in electricity markets vol. 1: Springer, 2010. Sotavento Wind Farm [Online]. Available: http://www.sotaventogalicia.com/.

[19] N. Amjady, J. Aghaei, and H. A. Shayanfar, "Stochastic multiobjective market clearing of joint energy and reserves auctions ensuring power system security," IEEE Transactions on Power Systems, vol. 24, pp. 1841-1854, 2009.

[20] Red Eléctrica de España, e-sios [Online]. Available: http://www.esios.ree.es/web-publica. 